\title{
Prevalence and burden of illness of treated hemolytic neonatal hyperbilirubinemia in a privately insured population in the United States
}

Tzy-Chyi Yu ${ }^{1}$, Chi Nguyen ${ }^{2 *}$, Nancy Ruiz ${ }^{1}$, Siting Zhou ${ }^{2}$, Xian Zhang ${ }^{2}$, Elaine A. Böing ${ }^{1}$ and Hiangkiat Tan ${ }^{2}$

\begin{abstract}
Background: Prevalence of hemolytic neonatal hyperbilirubinemia (NHB) is not well characterized, and economic burden at the population level is poorly understood. This study evaluated the prevalence, clinical characteristics, and economic burden of hemolytic NHB newborns receiving treatment in U.S. real-world settings.

Methods: This cohort study used administrative claims from 01/01/2011 to 08/31/2017. The treated cohort had hemolytic NHB diagnosis and received phototherapy, intravenous immunoglobulin, and/or exchange transfusions. They were matched with non-NHB newborns who had neither NHB nor related treatments on the following: delivery hospital/area, gender, delivery route, estimated gestational age (GA), health plan eligibility, and closest date of birth within 5 years. Inferential statistics were reported.

Results: The annual NHB prevalence was 29.6 to 31.7\%; hemolytic NHB, 1.8 to 2.4\%; treated hemolytic NHB, 0.46 to $0.55 \%$, between 2011 and 2016. The matched analysis included 1373 pairs $\geq 35$ weeks GA. The treated hemolytic $\mathrm{NHB}$ cohort had significantly more birth trauma and hemorrhage (4.5\% vs. $2.4 \%, p=0.003)$, vacuum extractor affecting newborn ( $1.9 \%$ vs. $0.8 \%, p=0.014)$, and polycythemia neonatorum $(0.8 \%$ vs. $0 \%, p=0.001)$ than the matched non-NHB cohort. The treated hemolytic NHB cohort also had significantly longer mean birth hospital stays (4.5 vs. 3.0 days, $p<0.001)$, higher level $2-4$ neonatal intensive care admissions $(15.7 \%$ vs. $2.4,15.9 \%$ vs. 2.8 and $10.6 \%$ vs. $2.5 \%$, respectively, all $p<0.001)$ and higher 30 -day readmission ( $8.7 \%$ vs. $1.7 \%, p<0.001)$.

One-month and one-year average total costs of care were significantly higher for the treated hemolytic NHB cohort vs. the matched non-NHB cohort, $\$ 14,405$ vs. $\$ 5527(p<0.001)$ and $\$ 21,556$ vs. $\$ 12,986(p<0.001)$, respectively. The average costs for 30 -day readmission among newborns who readmitted were $\$ 13,593$ for the treated hemolytic NHB cohort and $\$ 3638$ for the matched non-NHB cohort, $p<0.001$. The authors extrapolated GA-adjusted prevalence of treated hemolytic NHB in the U.S. newborn population $\geq 35$ weeks GA and estimated an incremental healthcare expenditure of $\$ 177.0$ million during the first month after birth in 2016.

Conclusions: The prevalence of treated hemolytic NHB was 4.6-5.5 patients per 1000 newborns. This high-risk hemolytic NHB imposed substantial burdens of healthcare resource utilization and incremental costs on newborns, their caregivers, and the healthcare system.
\end{abstract}

Keywords: Hemolytic neonatal hyperbilirubinemia, Neonatal hyperbilirubinemia, Prevalence, Clinical characteristics, Healthcare resource utilization, Costs, Burden of illness

\footnotetext{
* Correspondence: cnguyen@healthcore.com

${ }^{2}$ HealthCore, Inc., An Independent Subsidiary of Anthem, Inc, Wilmington, DE

19801, USA

Full list of author information is available at the end of the article
}

(c) The Author(s). 2019 Open Access This article is distributed under the terms of the Creative Commons Attribution 4.0 International License (http://creativecommons.org/licenses/by/4.0/), which permits unrestricted use, distribution, and reproduction in any medium, provided you give appropriate credit to the original author(s) and the source, provide a link to the Creative Commons license, and indicate if changes were made. The Creative Commons Public Domain Dedication waiver (http://creativecommons.org/publicdomain/zero/1.0/) applies to the data made available in this article, unless otherwise stated. 


\section{Background}

Neonatal hyperbilirubinemia (NHB), a common condition in newborn infants, results from elevated blood bilirubin levels. The excessive bilirubin manifests as yellowing of the skin and the normally white outer layer of the eyeballs [1-3]. While most cases resolve quickly without intervention, NHB is a common reason for inpatient readmissions, and admission to the neonatal intensive care unit (NICU) $[4,5]$. The prevalence of NHB is not precisely known, however, estimates suggest that approximately $50 \%$ full-term and $80 \%$ preterm [6] newborns develop some form of NHB. High-risk NHB occurs in $8-9 \%$ of neonates during the first week after birth $[5,7]$.

The origin of NHB may be physiologic or pathologic. Physiologic NHB may be caused by neonate immaturity and the resulting inability to cope with elevated levels of bilirubin [8]. This benign form resolves itself in 2-3 weeks following birth, and usually without treatment $[1,2]$. Pathologic NHB may be caused by hemolytic disease of the newborn (HDN), red blood cell (RBC) enzyme deficiency, or impaired bilirubin excretion [9]. HDN results from incompatibilities between maternal and fetal blood types ( $\mathrm{hh}$, $\mathrm{ABO}$ or a minor blood group), which may cause ruptures in fetal RBCs and elevated bilirubin levels. Hemolytic NHB usually appears within $24 \mathrm{~h}$ after birth [1, 2, 4].

The American Academy of Pediatrics (AAP) clinical practice guidelines address the assessment, screening, and treatment of NHB among infants at $\geq 35$ weeks of gestation [10]. Risk assessment and treatment nomograms based on total serum bilirubin level, postnatal age in hours, and gestational age of the newborn with the presence or absence of risk factors are available to guide patient management [10]. Similar guidelines are not available for neonates at less than 35 weeks of gestation because of scant evidence-based data, differences in clinical manifestations and unclear treatment outcomes [11].

When treatment is indicated, AAP guidelines recommend phototherapy as the initial treatment $[6,10]$. In cases where bilirubin levels continue to increase despite phototherapy, the guidelines recommend adding exchange transfusion of whole blood to the treatment regimen, typically in the $\operatorname{NICU}[2,10]$. For hemolytic cases, AAP guidelines recommend the administration of intravenous immunoglobulin (IVIg) as adjunctive therapy when bilirubin levels continue to rise despite intensive phototherapy $[2,10]$. These challenges in management of high-risk hyperbilirubinemia substantially increase the urgency for safer and more effective screening and/or treatment options, especially when viewed against the knowledge that the permanent sequelae of kernicterus spectrum disorders (KSDs) might be prevented.
To the best of our knowledge, the prevalence of hemolytic NHB newborns receiving treatment has not been well characterized, and economic burden at the population level is poorly understood. Our study aimed to address this knowledge gap. We focused on newborns with hemolytic NHB who received treatment because the receipt of intervention indicated that those neonates met the AAP guideline for the recommendation of intervention in order to prevent severe NHB and the spectrum of associated complications [10, 12-14].

\section{Methods \\ Design and data source}

This retrospective matched cohort study used the HealthCore Integrated Research Database (HIRD ${ }^{\mathrm{SM}}$ ), a geographically dispersed managed-care repository with claims data on more than 45 million enrollees residing across all 50 states, to identify infants born from $01 / 01 / 2011$ through $08 / 31 / 2017$. The HIRD is one of the largest privately insured population databases in the U.S [15]. This observational study was exempt from informed consent stipulations as researchers accessed a limited data set without individual enrollee identifiers and only summary statistics were reported. The study complied with all relevant provisions of the Health Insurance Portability and Accountability Act.

\section{Study population}

Newborns were linked to their birth mothers via shared health plan subscriber identification (ID) numbers. Mothers' delivery dates were verified within 32 days of newborns' dates of birth using delivery codes (Appendix: Table 7). Infants with 30-day or longer continuous enrollment after birth and mothers with at least 12 months of continuous health plan enrollment before delivery were included. All newborns, regardless of their estimated gestational age (GA), were included for NHB prevalence estimation. The treated hemolytic NHB and matched non-NHB cohorts were selected among newborns $\geq 35$ weeks GA. We excluded newborns $<35$ weeks GA as there was no clinical practice guidelines available for this group due to lack of evidence-based data, variabilities in clinical manifestations, and uncertainties about treatment benefits [11].

NHB population was defined as newborns with $\geq 1$ International Classification of Diseases (ICD)-9/10-CM diagnostic codes of NHB (ICD-9-CM $=773.0,773.1$, 773.2, 773.4, 774.x and ICD-10-CM = P55.x, P57.x, P58.x, P59.x) during the first 30 days after birth. Newborns with $\geq 1$ ICD-9/10-CM diagnosis codes of ICD-9-CM = 773.0, 773.1， 773.2，773.4， 774.0, 774.1, 774.7 and ICD-10-CM = P55.x, P57.x, P58.0, P58.1, 
P58.8, P58.9 were selected for the population of NHB with hemolysis indicators or hemolytic NHB (Appendix: Table 8).

\section{Treated hemolytic NHB cohort}

Treated hemolytic NHB cohort were selected from the hemolytic NHB population if they were $\geq 35$ weeks GA and received at least one NHB intervention including: phototherapy (Healthcare Common Procedure Coding System $[\mathrm{HCPCS}]=\mathrm{E} 0202, \quad$ S9098; $\mathrm{ICD}-9-\mathrm{CM}$ procedure $=99.83$; ICD-10-PCS $=6 \mathrm{~A} 600 \mathrm{ZZ}, 6 \mathrm{A601ZZ})$, IVIg treatment along with NHB diagnosis code on the same claim $(\mathrm{CPT}=90283$, 90284; Generic Product Identifier [GPI] $=19100020 x ; \quad$ HCPCS $=$ J1459, J1556, J1557, J1559, J1561, J1562, J1566, J1568, J1569, J1572, J1599), or exchange transfusions $(\mathrm{CPT}=36450,36456$; ICD-9-CM procedure $=99.01 ; \mathrm{ICD}-10-\mathrm{PCS}=30233 \mathrm{H} 1,30243 \mathrm{H} 1)$.

\section{Non-NHB cohort}

A non-NHB cohort was established using 1:1 matching with newborns in the treated hemolytic NHB cohort $\geq 35$ weeks GA. Inclusion in the non-NHB cohort required the absence of NHB diagnostic codes, no NHB treatment and a minimum of 30-day health plan enrollment after birth. Exact matching was performed based on delivery hospital/provider, gender, delivery route (C-section or vaginal), estimated GA, and post-index health plan continuous enrollment. When the matching of delivery hospital/provider was not possible, residence zip code (5-digit) was used instead. After all factors of interest were matched, newborns with the closest date of birth within 5 years were selected.

\section{Gestational age}

We calculated the GA of a newborn from prenatal procedure testing dates, from a range of common prenatal tests in the mother's medical claims, using the weighted procedure date-based average methodology, as described by Wallace et al. [16]. This method demonstrated that $67 \%$ of all deliveries and $60 \%$ of preterm deliveries had estimated GA staying within one week of the actual GA [16].

\section{Outcomes}

\section{Prevalence of NHB}

The annual prevalence of NHB, hemolytic NHB and treated hemolytic NHB were estimated for 2011 through 2016 as the number of newborns diagnosed with a disease divided by the total number of newborns after mother-infant linkage and health plan eligibility requirement during a particular calendar year.

\section{Hospitalization and healthcare resource utilization}

All-cause hospital measures included birth hospitalization, length of stay, NICU admissions, receipt of NHB treatments, and readmissions. The use of emergency department (ED) visits, physician office visits, other outpatient visits, and prescription fills were also presented. All healthcare resource utilization during the first 30 days and first year after birth were summarized.

\section{Clinical characteristics and outcomes}

The effects of hyperbilirubinemia on the brain and neurodevelopmental status were examined by evaluating occurrences of kernicterus, cerebral palsy, encephalopathy, hearing and vision loss, motor dysfunction, and neurodevelopmental delay during the first year after birth. These clinical outcomes were identified using ICD-9/ 10 -CM diagnosis codes, requiring $\geq 1$ diagnosis for inpatient/ED settings or $\geq 2$ diagnoses on distinct dates for physician office settings (Appendix: Table 9).

\section{Costs of care}

Total all-cause costs during the first 30 days and first year after birth were reported. Since newborn care during birth hospitalization could be billed under their mothers' plan ID, mothers' delivery hospitalization costs were included to avoid any potential unequal underestimation between the newborn cohorts. These costs were the sum of the total paid amount by health plans, members' out-of-pocket costs, and coordination of benefits. Total costs consisted of expenses incurred in inpatient, ED, office visits, other outpatient settings and pharmacy costs. Costs were adjusted for inflation using the Medical Care Consumer Price Index, and calculated in terms of 2017 U.S. dollars [17].

\section{Extrapolation to the U.S. newborn population}

Using U.S. Centers for Disease Control and Prevention (CDC) 2016 birth data by gestational age [18] and the estimated prevalence from our study, we applied a direct standardization method to extrapolate the 2016 U.S. GA-adjusted treated hemolytic NHB prevalence [19]. We then calculated population-level total healthcare expenditure based on our extrapolated prevalence and costs estimates.

\section{Statistical analysis}

All outcome measures were compared between the treated hemolytic NHB and matched non-NHB cohorts. Statistical differences between groups were assessed using McNemar or McNemar-Bowker tests for categorical variables and paired t-tests or Wilcoxon signed-rank tests for continuous variables, respectively. A conventional alpha of 0.05 with two-tailed level of significance was used to interpret statistical significance. Statistical 
analyses were performed with SAS EG 7.1 (SAS Institute, Cary, NC).

\section{Results}

\section{Study population}

Of the 1.4 million identified newborns, 365,937 were successfully linked to their birth mothers (Fig. 1). A total of 1673 newborns with hemolytic NHB received treatment and were of $\geq 35$ weeks GA. Among those, 1373 treated hemolytic NHB newborns were matched with non-NHB newborns; the matching rate was $82.1 \%$.

\section{Prevalence of NHB}

The annual prevalence of NHB ranged from 29.6 to $31.7 \%$ during 2011 to 2016. The prevalence of hemolytic NHB during that period ranged from 1.8 to $2.4 \%$, while the range for treated hemolytic NHB was 0.46 to $0.55 \%$ (Fig. 2). Upon stratification by estimated GA, the prevalence (95\% Confidence Interval (CI)) of NHB among newborns < 35 weeks GA was 49.4\% (95\% CI: 48.6-50.1\%), $38.4 \%$ (95\% CI: 37.9-38.8\%) of those 35-37 weeks GA, and $27.9 \%$ (95\% CI: $27.7-28.1 \%$ ) of those $>37$ weeks GA during 2011 to 2016. Hemolytic NHB was reported in $2.8 \%$ (95\% CI: $2.5-3.0 \%$ ) of newborns < 35 weeks GA, 2.3\% (95\% CI: $2.1-2.4 \%)$ of those $35-37$ weeks GA, and $2.0 \%$ (95\% CI: $1.9-2.0 \%$ ) of those $>37$ weeks GA. The prevalence of treated hemolytic NHB among newborns < 35 weeks GA was $1.09 \%$ (95\% CI: $0.93-1.25 \%$ ), $0.70 \%$ (95\% CI: $0.62-0.77 \%$ ) of those $35-37$ weeks GA, and $0.44 \%$ (95\% CI: $0.41-0.46 \%$ ) of those $>37$ weeks GA (Table 1).

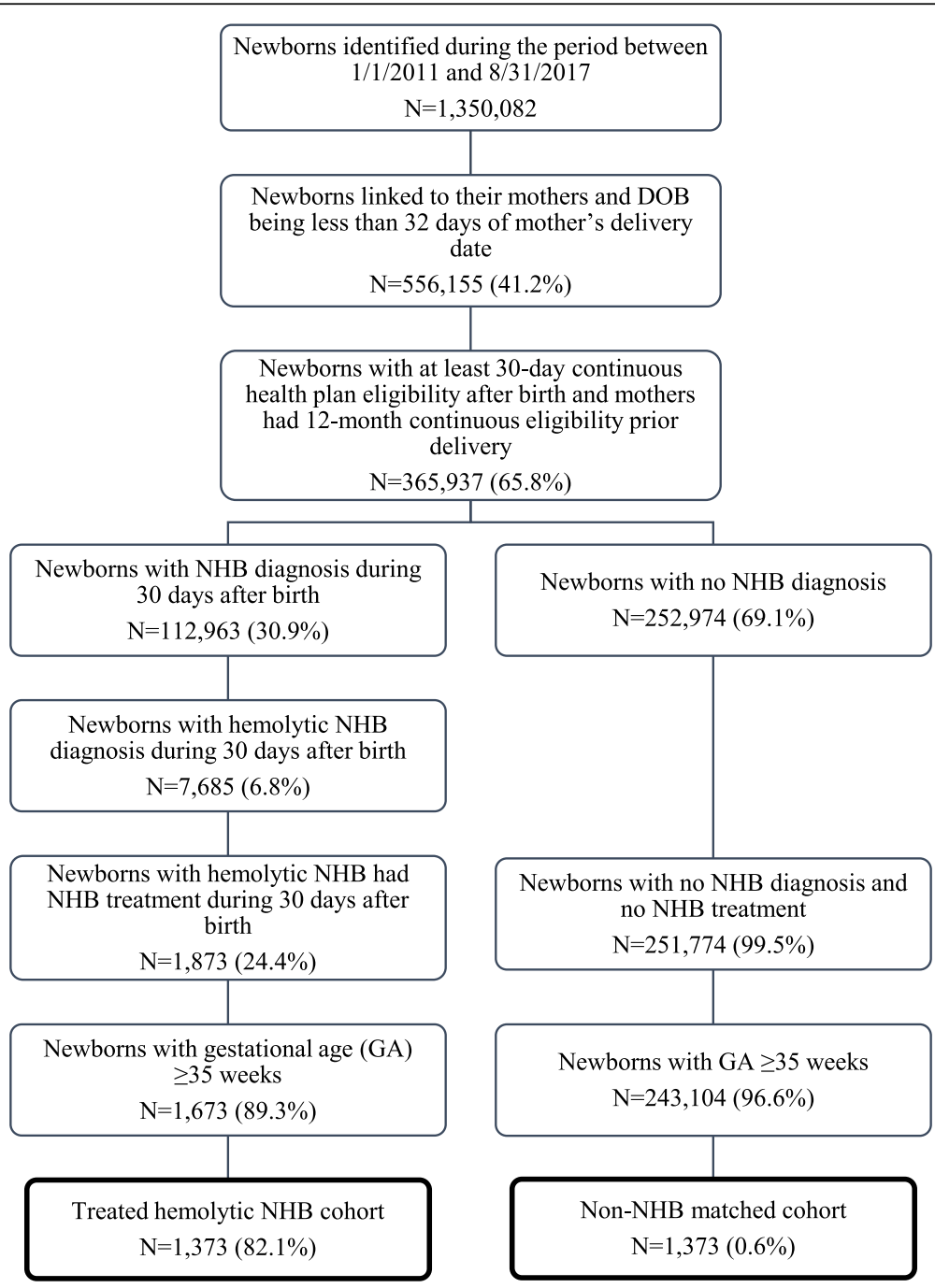

Fig. 1 Flow chart of the study population. Treated hemolytic NHB newborns were exactly matched to non-NHB newborns on delivery hospital/ provider, gender, delivery route (Csection or vaginal), estimated GA, and post-index health plan continuous enrollment. When the matching of delivery hospital/provider was not possible, residence zip code (5-digit) was used instead. After all of the above factors were matched, newborn with the closest DOB within 5 years was selected. DOB: date of birth; GA: gestational age; NHB: neonatal hyperbilirubinemia 


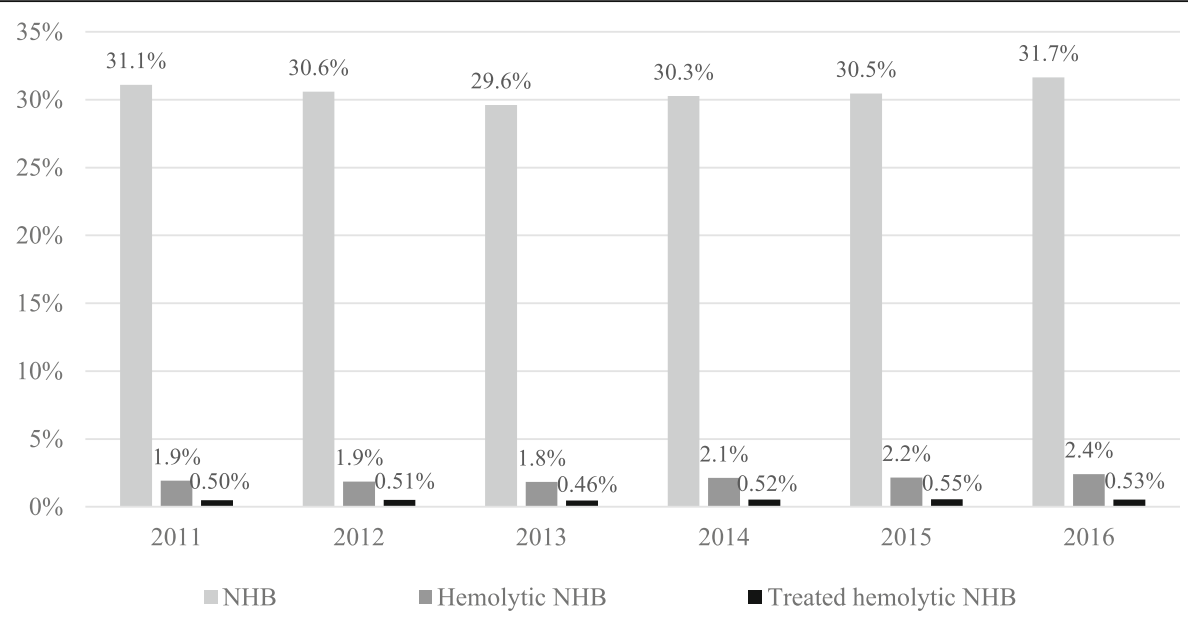

NHB: neonatal hyperbilirubinemia

Fig. 2 Prevalence of NHB, hemolytic NHB and treated hemolytic NHB stratified by GA from 2011 to 2016

\section{Mother and newborn demographic and clinical characteristics}

The mean age of mothers of treated hemolytic NHB and matched non-NHB (32.2 vs. 32.1 years, $p=0.40$ ), region of residence, type of health plan, comorbidity and gestational diabetes were similar at time of delivery. Slightly less than one-third (29.1\%) of births was delivered by C-section, and $18.2 \%$ of newborns were of $35-37$ weeks GA in each cohort (Table 2).

\section{NHB treatment}

During birth hospitalizations, $69.1 \%$ of the treated hemolytic NHB cohort received treatment. During the first 30 days after birth, $98.9 \%$ received phototherapy only, $0.3 \%$ received exchange transfusion only, $0.1 \%$ received phototherapy plus IVIg, and $0.7 \%$ received phototherapy plus exchange transfusion (Table 3 ).

\section{Newborn clinical conditions and neurodevelopmental disorders}

Newborns in the treated hemolytic NHB cohort had significantly higher proportions of birth trauma and hemorrhage $(4.5 \%$ vs. $2.4 \%, p=0.003)$, delivery by vacuum extractor affecting newborn $(1.9 \%$ vs. $0.8 \%, p=0.014)$, and polycythemia neonatorum $(0.8 \%$ vs. $0.0 \%, p=0.001)$ compared to the matched non-NHB cohort (Table 4). No difference was observed in neurodevelopmental disorders during the first year after birth between cohorts. Nine $(1.2 \%)$ of the treated hemolytic NHB newborns had kernicterus.

\section{Healthcare resource utilization and costs during $\mathbf{3 0}$ days after birth}

Treated hemolytic NHB newborns had longer average length of stay during birth hospitalization (4.5 days vs. 3.0 days; $p<0.001$ ), and a greater proportion were admitted to NICU $(82.6 \%$ vs. $70.0 \%$; p $<0.001)$ compared to matched non-NHB newborns (Table 5). Significantly higher proportions of treated hemolytic NHB newborns were admitted to NICU levels 2-4 ( $15.7 \%$ vs. $2.4 \%$; $15.9 \%$ vs. $2.8 \%$; and $10.6 \%$ vs. $2.5 \%$, respectively; all $p<0.001)$. Hospital readmissions and physician office visits were significantly higher for treated hemolytic NHB newborns than the matched non-NHB cohort, $8.7 \%$ vs. $1.7 \%(p<0.001)$ and $90.8 \%$

Table 1 Prevalence of NHB, hemolytic NHB and treated hemolytic NHB stratified by gestational age (GA) from 2011 to 2016

\begin{tabular}{|c|c|c|c|}
\hline \multirow[t]{2}{*}{ Estimated GA } & \multicolumn{3}{|c|}{ Prevalence (95\% Confidence Interval) } \\
\hline & $\mathrm{NHB}$ & Hemolytic NHB & Treated hemolytic NHB \\
\hline$>37$ weeks GA & $27.9 \%(27.7-28.1 \%)$ & $2.0 \%(1.9-2.0 \%)$ & $0.44 \%(0.41-0.46 \%)$ \\
\hline 35-37 weeks GA & $38.4 \%(37.9-38.8 \%)$ & $2.3 \%(2.1-2.4 \%)$ & $0.70 \%(0.62-0.77 \%)$ \\
\hline$<35$ weeks GA & $49.4 \%(48.6-50.1 \%)$ & $2.8 \%(2.5-3.0 \%)$ & $1.09 \%(0.93-1.25 \%)$ \\
\hline All newborns & $30.6 \%(30.5-30.8 \%)$ & $2.0 \%(2.0-2.1 \%)$ & $0.51 \%(0.49-0.54 \%)$ \\
\hline
\end{tabular}


Table 2 Mother and newborn demographic and clinical characteristics

\begin{tabular}{|c|c|c|c|}
\hline & $\begin{array}{l}\text { Treated } \\
\text { hemolytic } \\
\text { NHB cohort } \\
(N=1,373) \\
\end{array}$ & $\begin{array}{l}\text { Matched } \\
\text { non-NHB } \\
\text { cohort } \\
(N=1,373) \\
\end{array}$ & $p$-value ${ }^{3}$ \\
\hline \multicolumn{4}{|l|}{ Mothers } \\
\hline $\begin{array}{l}\text { Age on delivery (year), } \\
\text { mean (SD) }\end{array}$ & $32.2(4.63)$ & $32.1(4.43)$ & 0.401 \\
\hline Geographic region, $n(\%)$ & & & 0.394 \\
\hline Northeast & $289(21.0)$ & $285(20.8)$ & \\
\hline Midwest & $503(36.6)$ & $494(36.0)$ & \\
\hline South & $363(26.4)$ & $368(26.8)$ & \\
\hline West & $212(15.4)$ & $218(15.9)$ & \\
\hline Other/Unknown ${ }^{1}$ & $6(0.4)$ & $8(0.6)$ & \\
\hline Health Plan type, $n(\%)$ & & & 0.928 \\
\hline $\mathrm{HMO}$ & $270(19.7)$ & $262(19.1)$ & \\
\hline PPO & $831(60.5)$ & $845(61.5)$ & \\
\hline $\mathrm{CDHP}$ & $272(19.8)$ & $266(19.4)$ & \\
\hline $\begin{array}{l}\text { Modified Deyo-Charlson } \\
\text { Comorbidity Index } \\
\text { mean (SD) }\end{array}$ & $0.1(0.47)$ & $0.1(0.40)$ & 0.274 \\
\hline Gestational diabetes, $n(\%)$ & $239(17.4)$ & $222(16.2)$ & 0.367 \\
\hline C-section, $n(\%)$ & $399(29.1)$ & $399(29.1)$ & - \\
\hline \multicolumn{4}{|l|}{ Newborns } \\
\hline Gender, $n(\%)$ & & & - \\
\hline Male & $667(48.6)$ & $667(48.6)$ & \\
\hline Female & $706(51.4)$ & $706(51.4)$ & \\
\hline $\begin{array}{l}\text { Estimated gestational } \\
\text { age, } n(\%)\end{array}$ & & & - \\
\hline 35-37 weeks & $250(18.2)$ & $250(18.2)$ & \\
\hline$>37$ weeks & $1,123(81.8)$ & $1,123(81.8)$ & \\
\hline Year of birth, $n(\%)$ & & & $<0.001$ \\
\hline 2011 & $217(15.8)$ & $217(15.8)$ & \\
\hline 2012 & $211(15.4)$ & $195(14.2)$ & \\
\hline 2013 & $187(13.6)$ & $206(15.0)$ & \\
\hline 2014 & $197(14.3)$ & $239(17.4)$ & \\
\hline 2015 & $228(16.6)$ & $239(17.4)$ & \\
\hline 2016 & $208(15.1)$ & $188(13.7)$ & \\
\hline 2017 & $125(9.1)$ & $89(6.5)$ & \\
\hline
\end{tabular}

NHB neonatal hyperbilirubinemia, $S D$ standard deviation, $H M O$ Health Maintenance Organization, PPO Provider Preferred Organization, CDHP Consumer Driven Health Products

${ }^{1}$ Other/unknown region includes American Samoa, Guam, Northern Mariana Islands, Puerto Rico, Virgin Islands or unknown region

${ }^{2}$ Modified Deyo-Charlson Comorbidity Index was estimated using ICD-9/10-CM codes by Beyrer et al. [36]

${ }^{3} \mathrm{p}$-value calculated using McNemar test or McNemar-Bowker test for categorical variables and paired t-test or Wilcoxon signed-rank test for continuous variables
Table 3 NHB treatment pattern during 30 days after birth

\begin{tabular}{ll}
\hline Treatment pattern & $\begin{array}{l}\text { Treated hemolytic } \\
\text { NHB cohort } \\
(N=1373)\end{array}$ \\
\hline $\begin{array}{l}\text { During birth hospitalization (mutually exclusive) } \\
\text { Any NHB treatment during birth hospitalization, }\end{array}$ & $949(69.1)$ \\
$n \%$ & $937(68.2)$ \\
Phototherapy only, $n \%$ & $0(0)$ \\
IVlg only, $n \%$ & $4(0.3)$ \\
Exchange transfusion only, n\% & $2(0.1)$ \\
Phototherapy + IVlg, $n \%$ & $6(0.4)$ \\
Phototherapy + Exchange transfusion, $n \%$ & $0(0)$ \\
IVlg + Exchange transfusion, $n \%$ & $0(0)$ \\
Phototherapy + IVlg + Exchange transfusion, $n \%$ & \\
During 30 days after birth (mutually exclusive) & \\
Phototherapy only, $n \%$ & $1358(98.9)$ \\
IVlg only, $n \%$ & $0(0)$ \\
Exchange transfusion only, n\% & $4(0.3)$ \\
Phototherapy + IVlg, $n \%$ & $2(0.1)$ \\
Phototherapy + Exchange transfusion, $n \%$ & $9(0.7)$ \\
IVlg + Exchange transfusion, $n \%$ & $0(0)$ \\
Phototherapy + IVlg + Exchange transfusion, $n \%$ & $0(0)$ \\
\hline
\end{tabular}

NHB neonatal hyperbilirubinemia, $I V I g$ intravenous immunoglobulin

vs. $82.6 \%(\mathrm{p}<0.001)$, respectively. No difference was reported for ED visits $(1.7 \%$ vs. $1.4 \%, p=0.54)$ and prescription fills $(6.3 \%$ vs. $6.0 \%, p=0.81)$ between the groups.

Mean (SD) total 30-day all-cause costs for the newborns were $\$ 14,405(\$ 43,918)$ for the treated hemolytic NHB group and $\$ 5,527(\$ 50,079)$ for the matched non-NHB cohort $(\mathrm{p}<0.001)$. The treated hemolytic NHB group incurred mean (SD) total inpatient hospitalization costs of $\$ 13,794 \quad(\$ 43,949)$ compared to $\$ 5,216(\$ 50,083)$ in the matched non-NHB group, $p<$ 0.001. The average costs of readmissions among those readmitted to the hospitals were $\$ 13,593(\$ 34,524)$ and $\$ 3,638 \quad(\$ 5,685)$ for the treated hemolytic NHB and non-NHB groups, respectively. The mean (SD) 30-day incremental total all-cause costs associated with treated hemolytic NHB newborns was $\$ 9,381(\$ 63,558)$ composed of $\$ 8,878 \quad(\$ 59,943)$ from newborns plus $\$ 503(\$ 19,969)$ from mothers' delivery hospitalization.

\section{Healthcare resource utilization and costs during one year after birth}

Of 1,373 pairs, 765 (55.7\%) matched pairs with one-year follow-up were included in the analysis. There was no statistically significant difference between the two 
Table 4 Newborn clinical conditions and neurodevelopmental disorders

\begin{tabular}{|c|c|c|c|}
\hline & $\begin{array}{l}\text { Treated } \\
\text { hemolytic } \\
\text { NHB cohort }\end{array}$ & $\begin{array}{l}\text { Matched } \\
\text { non-NHB } \\
\text { cohort }\end{array}$ & $p$-value \\
\hline $\begin{array}{l}\text { Clinical conditions during } \\
30 \text { days after birth, total } n\end{array}$ & 1,373 & 1,373 & \\
\hline $\begin{array}{l}\text { Breech delivery and } \\
\text { extraction affecting } \\
\text { fetus or newborn, } n(\%)\end{array}$ & $114(8.3)$ & $107(7.8)$ & 0.579 \\
\hline $\begin{array}{l}\text { Birth trauma and } \\
\text { hemorrhage, } n(\%)\end{array}$ & $62(4.5)$ & $33(2.4)$ & 0.003 \\
\hline $\begin{array}{l}\text { Delivery by vacuum } \\
\text { extractor affecting fetus } \\
\text { or newborn, } n(\%)\end{array}$ & $26(1.9)$ & $11(0.8)$ & 0.014 \\
\hline $\begin{array}{l}\text { Polycythemia neonatorum, } \\
n(\%)\end{array}$ & $11(0.8)$ & $0(0)$ & 0.001 \\
\hline $\begin{array}{l}\text { Other malpresentation, } \\
\text { malposition, and disproportion } \\
\text { during labor and delivery } \\
\text { affecting fetus or newborn, } \\
n(\%)\end{array}$ & $9(0.7)$ & $9(0.7)$ & 1.000 \\
\hline $\begin{array}{l}\text { Forceps delivery affecting } \\
\text { fetus or newborn, } n(\%)\end{array}$ & $5(0.4)$ & $7(0.5)$ & 0.564 \\
\hline $\begin{array}{l}\text { Neonatal hematemesis and } \\
\text { melena due to swallowed } \\
\text { maternal blood, } n(\%)\end{array}$ & $0(0)$ & $0(0)$ & - \\
\hline $\begin{array}{l}\text { Neurodevelopmental disorders } \\
\text { during one year after birth, } \\
\text { total } n\end{array}$ & 765 & 765 & \\
\hline Kernicterus, $n(\%)$ & $9(1.2)$ & $0(0)$ & 0.004 \\
\hline Motor dysfunction, $n(\%)$ & $4(0.5)$ & $2(0.3)$ & 0.687 \\
\hline Hearing loss, $n(\%)$ & $3(0.4)$ & $2(0.3)$ & 1.000 \\
\hline Encephalopathy, n (\%) & $2(0.3)$ & $2(0.3)$ & 1.000 \\
\hline Abnormal behavior, $n(\%)$ & $1(0.1)$ & $3(0.4)$ & 0.625 \\
\hline Cerebral palsy, $n$ (\%) & $1(0.1)$ & $0(0)$ & 1.000 \\
\hline Vision loss, n (\%) & $0(0)$ & $2(0.3)$ & 0.500 \\
\hline $\begin{array}{l}\text { Neurodevelopmental } \\
\text { delay, } n(\%)\end{array}$ & $0(0)$ & $1(0.1)$ & 1.000 \\
\hline Cognitive disorders, $n(\%)$ & $0(0)$ & $0(0)$ & - \\
\hline Language disorders, $n(\%)$ & $0(0)$ & $0(0)$ & _- \\
\hline
\end{tabular}

NHB neonatal hyperbilirubinemia

${ }^{1} p$-values calculated using McNemar test or Fisher's exact test for binary variables

cohorts in inpatient admissions and ED visits during the period from 31 days to 1 year after birth. Physician office visits and prescription fills were slightly higher in the treated hemolytic NHB group compared to the matched non-NHB group $(99.7 \%$ vs. $97.4 \%, p<0.001$ and $69.7 \%$ vs. $63.5 \%, p=0.009$, respectively). The mean (SD) total one-year all-cause costs incurred by the treated hemolytic NHB cohort were \$21,556 $(\$ 60,823)$ compared to $\$ 12,986(\$ 72,164)$ in the matched non-NHB cohort, $p<0.001$. The average (SD) one-year incremental total all-cause costs associated with treated hemolytic NHB was $\$ 9,383$ (\$84,478), consisting of $\$ 813(\$ 12,922)$ from mother's delivery hospitalization and $\$ 8,570$ $(\$ 82,379)$ from newborns (Table 6).

\section{Extrapolation to the U.S. population}

The extrapolation of 2016 U.S. GA-adjusted treated hemolytic NHB prevalence was $0.53 \%, 20,854$ newborns (95\% CI, 18,398-23,311) among 3.9 million newborns in the U.S. in 2016. Among newborns $\geq 35$ weeks GA, the GA-adjusted prevalence of treated hemolytic NHB was $0.50 \%$ resulting in 18,872 newborns (95\% CI, 16,523 - 21,221). The 18,872 treated hemolytic NHB newborns represent an estimated total healthcare expenditure of \$271.9 million and incremental costs of $\$ 177.0$ million compared with their counterparts without NHB during the first month after birth in the U.S. in 2016.

\section{Discussion}

To the best of our knowledge, this is the first study to estimate the prevalence of high-risk hemolytic NHB newborns receiving intervention, and to quantify the burden of hemolytic NHB in the US. The proportions of newborns with hemolytic NHB who received treatment were 0.46 to $0.55 \%$ in a privately insured population in the US. Although not prevalent, those high-risk hemolytic NHB neonates who received treatment were associated with substantial healthcare resource utilization and incremental economic burden.

NHB research in the U.S. has been limited, and prevalence estimates vary markedly in the handful of studies in the literature. In a systematic review that included 14 studies to examine the effects and outcomes of phototherapy, Woodgate and Jardine noted that about $50 \%$ of full-term and $80 \%$ preterm newborns developed jaundice [6]. In a survey at medical centers that practiced universal pre-discharge total serum bilirubin (TSB) screening, Bhutani et al. reported jaundice in $84 \%$ of healthy newborns $\geq 35$ weeks GA [20]. Another study, which used inpatient data from the Healthcare Costs and Utilization Project (HCUP), reported $15.6 \%$ of newborns had jaundice [21]. These variations could, in part, be due to differences in the study population, case definitions (e.g., TSB level vs. visible jaundice), data sources, and underdiagnosis or underreporting of mild cases. Mild NHB typically resolves without intervention, and may not be fully captured in administrative claims (used in our study) and hospital discharge data (HCUP). Such cases may not be reflected in reimbursements because of bundled payments, which could result in an underestimation of general NHB prevalence. 
Table 5 Healthcare resource utilization and costs during 30 days after birth

\begin{tabular}{|c|c|c|c|}
\hline & $\begin{array}{l}\text { Treated hemolytic NHB cohort } \\
(N=1373)\end{array}$ & $\begin{array}{l}\text { Matched non-NHB cohort } \\
(N=1373)\end{array}$ & $p$-value \\
\hline \multicolumn{4}{|l|}{ All-cause healthcare resource use } \\
\hline \multicolumn{4}{|l|}{ Inpatient } \\
\hline Birth hospitalization LOS, mean (SD) & $4.5(6.06)$ & $3.0(5.74)$ & $<0.001$ \\
\hline NICU admission during birth hospitalization, $n$ (\%) & $1,134(82.6)$ & $961(70.0)$ & $<0.001$ \\
\hline NICU Level 1 & $903(65.8)$ & $910(66.3)$ & 0.713 \\
\hline NICU Level 2 & $215(15.7)$ & $33(2.4)$ & $<0.001$ \\
\hline NICU Level 3 & $218(15.9)$ & $38(2.8)$ & $<0.001$ \\
\hline NICU Level 4 & $146(10.6)$ & $35(2.5)$ & $<0.001$ \\
\hline Readmission within 30-days after birth, $n(\%)$ & $119(8.7)$ & $23(1.7)$ & $<0.001$ \\
\hline LOS, mean (SD) & $2.4(2.62)$ & $1.7(1.34)$ & 0.033 \\
\hline Emergency room visits, $n(\%)$ & $23(1.7)$ & $19(1.4)$ & 0.537 \\
\hline Number of visits, mean (SD) & $1.0(0.21)$ & $1.1(0.23)$ & 0.919 \\
\hline Physician office visits, $n$ (\%) & $1,247(90.8)$ & $1,134(82.6)$ & $<0.001$ \\
\hline Number of visits, mean (SD) & $2.8(1.49)$ & $2.2(1.15)$ & $<0.001$ \\
\hline Other outpatient visits ${ }^{2}, n(\%)$ & $1,001(72.9)$ & $427(31.1)$ & $<0.001$ \\
\hline Number of visits, mean (SD) & $3.8(3.29)$ & $1.5(1.26)$ & $<0.001$ \\
\hline Prescription fills, $n(\%)$ & $86(6.3)$ & $83(6.0)$ & 0.811 \\
\hline Number of fills, mean (SD) & $1.2(0.43)$ & $1.1(0.36)$ & 0.533 \\
\hline \multicolumn{4}{|l|}{ All-cause healthcare costs, mean (SD), 2017 USD } \\
\hline Medical costs & $\$ 14,403(\$ 43,918)$ & $\$ 5,524(\$ 50,078)$ & $<0.001$ \\
\hline Inpatient (including birth hospitalization) & $\$ 13,794(\$ 43,949)$ & $\$ 5,216(\$ 50,083)$ & $<0.001$ \\
\hline Birth hospitalization & $\$ 12,616(\$ 42,475)$ & $\$ 5,155(\$ 50,080)$ & $<0.001$ \\
\hline Readmission during 30 days after birth ${ }^{3}$ & $\$ 13,593(\$ 34,524)$ & $\$ 3,638(\$ 5685)$ & $<0.001$ \\
\hline Emergency department & $\$ 20(\$ 187)$ & $\$ 17(\$ 169)$ & 0.636 \\
\hline Physician office visit & $\$ 313(\$ 258)$ & $\$ 224(\$ 203)$ & $<0.001$ \\
\hline Other outpatient visits & $\$ 276(\$ 651)$ & $\$ 67(\$ 289)$ & $<0.001$ \\
\hline Pharmacy costs & $\$ 2(\$ 12)$ & $\$ 2(\$ 28)$ & 0.923 \\
\hline Total medical and pharmacy costs & $\$ 14,405(\$ 43,918)$ & $\$ 5,527(\$ 50,079)$ & $<0.001$ \\
\hline \multicolumn{4}{|l|}{ Incremental all-cause healthcare costs } \\
\hline Treated hemolytic NHB newborn incremental costs & $\$ 8,878(\$ 59,943)$ & & \\
\hline Mother's delivery incremental costs ${ }^{4}$ & $\$ 503(\$ 19,969)$ & & \\
\hline Total incremental costs & $\$ 9,381(\$ 63,558)$ & & \\
\hline
\end{tabular}

NHB neonatal hyperbilirubinemia, SD standard deviation, LOS length of stay

${ }^{1} \mathrm{p}$-values calculated using McNemar test for binary variables and Wilcoxon signed-rank test for continuous variables

${ }^{2}$ Other outpatient visits included durable medical equipment, imaging, medication \& related services, procedures, physician other services, tests and occupational, physical or speech therapy

${ }^{3}$ Readmission costs calculated among those who had readmission during the first 30 days after birth, including 119 newborns in treated hemolytic NHB cohort and 23 newborns in matched non-NHB cohort

${ }^{4} \mathrm{~A}$ newborn's care and treatment could be billed to his/her mother's plan during birth hospitalization; mother's incremental costs of delivery hospitalization were included

Our study focused on NHB specifically with etiology of hemolytic diseases, and we found that approximately $7 \%$ of the NHB cases were hemolytic NHB. Our estimated prevalence of treated hemolytic NHB (ranging from $0.46-0.55 \%$ ) was comparable to $<1 \%$ of significant hemolysis reported by Wagle and Deshpande [22]. Chang et al. estimated that about $6 \%$ of newborns $\geq 35$ weeks GA received phototherapy at Kaiser Permanente hospitals [23]. Using our estimate that $7 \%$ of the NHB newborns in this study had hemolytic NHB along with 
Table 6 Healthcare resource utilization and costs during one year after birth

\begin{tabular}{|c|c|c|c|}
\hline & $\begin{array}{l}\text { Treated hemolytic NHB cohort } \\
(N=765)\end{array}$ & $\begin{array}{l}\text { Matched non-NHB cohort } \\
(N=765)\end{array}$ & $p$-value \\
\hline \multicolumn{4}{|l|}{ All-cause healthcare resource use } \\
\hline \multicolumn{4}{|l|}{ Inpatient } \\
\hline Readmission within 30-days after birth, $n(\%)$ & $60(7.8)$ & $14(1.8)$ & $<0.001$ \\
\hline Inpatient admission from 31 days to one year after birth & $36(4.7)$ & $24(3.1)$ & 0.109 \\
\hline Emergency room visits, $n$ (\%) & $138(18.0)$ & $125(16.3)$ & 0.378 \\
\hline Number of visits, mean (SD) & $1.3(0.72)$ & $1.2(0.50)$ & 0.690 \\
\hline Physician office visits, $n$ (\%) & $763(99.7)$ & $745(97.4)$ & $<0.001$ \\
\hline Number of visits, mean (SD) & $12.2(5.44)$ & $10.7(4.92)$ & $<0.001$ \\
\hline Other outpatient visits ${ }^{2}, n(\%)$ & $763(99.7)$ & $736(96.2)$ & $<0.001$ \\
\hline Number of visits, mean (SD) & $11.3(7.25)$ & $8.3(7.28)$ & $<0.001$ \\
\hline Prescription fills, $n(\%)$ & $533(69.7)$ & $486(63.5)$ & 0.009 \\
\hline Number of fills, mean (SD) & $4.3(4.36)$ & $3.9(3.94)$ & 0.140 \\
\hline \multicolumn{4}{|l|}{ All-cause healthcare costs, mean (SD), 2017 USD } \\
\hline \multicolumn{4}{|l|}{ Newborns } \\
\hline Medical costs & $\$ 21,407(\$ 60,808)$ & $\$ 12,784(\$ 71,669)$ & $<0.001$ \\
\hline Inpatient (including birth hospitalization) & $\$ 16,679(\$ 58,723)$ & $\$ 8865(\$ 70,060)$ & $<0.001$ \\
\hline Emergency department & $\$ 279(\$ 850)$ & $\$ 235(\$ 749)$ & 0.199 \\
\hline Physician office visits & $\$ 1,443(\$ 847)$ & $\$ 1,248(\$ 864)$ & $<0.001$ \\
\hline Other outpatient visits & $\$ 3,006(\$ 4,096)$ & $\$ 2,436(\$ 3,712)$ & $<0.001$ \\
\hline Pharmacy costs & $\$ 149(\$ 359)$ & $\$ 202(\$ 1494)$ & 0.038 \\
\hline Total newborn medical and pharmacy costs & $\$ 21,556(\$ 60,823)$ & $\$ 12,986(\$ 72,164)$ & $<0.001$ \\
\hline \multicolumn{4}{|l|}{ Incremental all-cause healthcare costs } \\
\hline Treated hemolytic NHB newborn incremental costs & $\$ 8,570(\$ 82,379)$ & & \\
\hline Mother's delivery incremental costs ${ }^{3}$ & $\$ 813(\$ 12,922)$ & & \\
\hline Total incremental costs & $\$ 9,383(\$ 84,478)$ & & \\
\hline
\end{tabular}

NHB neonatal hyperbilirubinemia, SD standard deviation

${ }^{1} p$-values calculated using McNemar test for binary variables and Wilcoxon signed-rank test for continuous variables

${ }^{2}$ Other outpatient visits included durable medical equipment, imaging, medication \& related services, procedures, physician other services, lab tests and occupational, physical or speech therapy

${ }^{3} \mathrm{~A}$ newborn's care and treatment could be billed to his/her mother's plan during birth hospitalization; mother's incremental costs of delivery hospitalization were included

the assumption that all the newborns in the Chang et al. study had NHB, we inferred that approximately $0.42 \%$ of newborns in Chang et al. were phototherapy-treated hemolytic NHB - which is close to our estimate. Treatment rates could vary remarkably as treatment practice across hospitals/institutions differ in how cases are identified and when treatment should be initiated [24, 25]. Additionally, prior literature suggested that NHB patients could be under-treated. One U.S. study showed that only approximately half $(54 \%)$ of healthy term newborns for whom AAP clinical practice guidelines recommended phototherapy received treatment [26].

We found that treated hemolytic NHB newborns had significantly longer length of stay during their birth hospitalization, higher 30 -day readmission rates, higher
NICU use and slightly higher rates of physician office visits, compared to their matched counterparts. Length of stay of mothers' delivery hospitalizations were also slightly longer in the treated hemolytic NHB cohort (2.9 days vs 2.5 days, data not shown). These findings suggest significant burden to patients, their caregivers, and the healthcare system. Prior studies have shown that NHB was as major cause of readmission. Approximately half $(51 \%)$ of all readmissions occurring 2 weeks after birth were attributable to NHB [27]. The increase in physician office visits we reported was also consistent with available literature, which found that NHB was associated with increased parental awareness, and newborns receiving phototherapy had higher rates of outpatient visits [28]. 
We also found that hemolytic NHB newborns who received treatment incurred 2.6 times the average costs of their matched non-NHB counterparts during the first 30 days after birth. The majority of the incremental cost was derived from birth hospitalizations. Indirect costs associated with patients' and caregivers' quality of life as well as caregivers' loss of productivity could not be evaluated using claims data. As of now, no prior study has examined the economic burden of hemolytic NHB. One earlier study estimated the average cost of childbirth via vaginal or caesarian at $\$ 18,329$ or $\$ 27,866$, respectively, in a private health plan [29]. Those estimates were close to the average costs, $\$ 20,568$, of the sum of maternal delivery (mean $(\mathrm{SD})=\$ 15,413(\$ 20,010)$, data not shown) and newborn birth hospitalization $(\$ 5,155(\$ 50,080)$, Table 5$)$ in the non-NHB cohort in our study. Such comparability might warrant the representativeness and generalizability of our study results to other privately insured populations. In this study, we found that the majority of treated hemolytic NHB newborns received phototherapy. A total of 15 (1\%) newborns received IVIg or ET, which are recommended by AAP when bilirubin levels continue to rise despite intensive phototherapy. This group imposed even greater economic burden with average (SD) total one-month all-cause costs of $\$ 81,065$ $(\$ 133,767)$ (data not shown).

We extrapolated our findings to the entire U.S. newborn population in 2016. The extrapolation estimated total healthcare expenditure of $\$ 271.9$ million and incremental costs of $\$ 177.0$ million among 18,872 treated hemolytic NHB newborns as compared with their counterparts without NHB during the first month after birth. Our extrapolation assumed our estimates were applicable to the U.S. newborn population mainly insured by private insurance plans or Medicaid. This projection should be interpreted with caution as privately insured populations tend to have higher socioeconomic status and healthcare expenditures than the Medicaid population [30]. Further research in the Medicaid newborn population is warranted to examine our assumptions and estimates.

We did not observe significant difference in neurodevelopment delay, language disorders, motor dysfunction, cerebral palsy, abnormal behavior, encephalopathy, hearing and vision loss between treated hemolytic NHB newborns and the matched non-NHB cohort during the first year of birth. However, the observation period was likely too short as many of these conditions might not be identifiable nor noticeable in the first year of life. Kernicterus, a brain injury resulting from severe NHB, was found in nine newborns, approximately $1.2 \%$ of all treated hemolytic NHB newborns during the one-year follow up. Kernicterus has been reported from 1.0 to 3.7 cases per 100,000 live birth in the literature [31, 32], but these incidence rates were estimated for the general population in contrast to the high-risk hemolytic NHB population (treated) in this study. As hemolytic NHB was strongly correlated with higher incidences of birth trauma, polycythemia, and other subsequent morbidities which could also cause neurodevelopment disorders, neurodevelopment disorders in this population could be due to a combination of hemolytic NHB and other morbidities, rather than hemolytic NHB alone.

Effective management of high-risk hemolytic NHB is critical to reduce the impact of disease burden on patients, their caregivers, and the healthcare system. Several studies have investigated comprehensive approaches, such as pre-discharge bilirubin screening for all newborns [25], or the implementation of a standard pathway including treatment algorithms (e.g., requiring irradiance compliance to ensure consistent delivery of effective phototherapy) and education to increase awareness among clinicians [33]. These comprehensive approaches have demonstrated success in reducing costs, length of stay [33] and hospital readmission rates [25]. In addition, new treatment options are needed. For example, an investigational treatment stannsoporfin (SnMP, a heme oxygenase inhibitor) with or without phototherapy was studied for use in the management of NHB or hemolytic NHB $[34,35]$.

\section{Limitations}

Our results should be interpreted in light of certain limitations. Known risk factors such as family history, race and ethnicity, and breastfeeding status are not available in administrative claims data. Cases of mild NHB do not usually require intervention, and can be underdiagnosed and/or under-coded in administrative data leading to underestimation of NHB and hemolytic NHB. The use of phototherapy during hospitalization might not have been observed due to bundled payments and/or under-coding. Duration on phototherapy was also not captured. This study population was from a U.S. privately insured population, which may limit the generalizability of these results to other population segments, such as Medicaid.

\section{Conclusions}

This is likely the first study estimating the prevalence of newborns with hemolytic NHB who received intervention in the U.S. This high-risk population imposes a substantial burden of healthcare resource utilization and incremental costs on newborns, their caregivers, and the healthcare system. Effective management protocols and emerging new treatments may help to mitigate the overall burden of hemolytic NHB. 


\section{Appendix}

Table 7 Codes to identify delivery

\section{Codes to identify all deliveries}

CPT codes

Routine obstetric care including antepartum care, vaginal delivery (with or without episiotomy, and/or forceps) and postpartum care

Vaginal delivery only (with or without episiotomy and/or forceps)

Vaginal delivery only (with or without episiotomy and/or forceps), including postpartum care

External cephalic version, with or without tocolysis

Delivery of placenta (separate procedure)

Antepartum care only; 4-6 visits

Antepartum care only; 7+ visits

Routine obstetric care including antepartum care, vaginal delivery (with or without episiotomy, and/or forceps) and postpartum care, after previous cesarean delivery

Vaginal delivery only, after previous cesarean delivery (with or without episiotomy and/or forceps);

Vaginal delivery only, after previous cesarean delivery (with or without episiotomy and/or forceps); including postpartum care

Routine obstetric care including antepartum care cesarean delivery, and postpartum care

Cesarean delivery only

Cesarean delivery only; including postpartum care

Routine obstetric care including antepartum care, cesarean delivery, and postpartum care, following attempted vaginal delivery after previous cesarean delivery

Cesarean delivery only, following attempted vaginal delivery after previous cesarean delivery;

Cesarean delivery only, following attempted vaginal delivery after previous cesarean delivery; including postpartum care

Revenue Codes

Labor Room/Delivery

ICD-9-CM diagnosis

Outcome of delivery

Single liveborn

Twins, both liveborn

Twins, one liveborn one stillborn

Other multiple, all liveborn

Other multiple, some liveborn

Unspecified

Liveborn infants consuming healthcare

Single liveborn

Twin, mate liveborn

Twin, mate stillborn
Table 7 Codes to identify delivery (Continued)

Twin, unspecified

V33.xx

Other multiple, mates all liveborn

V34.xx

Other multiple, mates all stillborn

V36.xx

Other multiple, unspecified

V37.xx

Unspecified

V39.xx

Normal delivery

650.xx

Forceps or vacuum extractor delivery without

$669.5 x$ mention of indication

59425

59,426

59612

Breech extraction, without mention of indication

$669.6 x$

Cesarean delivery, without mention of indication

$669.7 x$

ICD-9-CM procedure

Forceps, vacuum, and breech delivery

72.xx

Other procedures inducing or assisting delivery 73.xx

Cesarean section and removal of fetus

$74 . x x$

ICD-10-CM diagnosis

Outcome of delivery

Single liveborn

Z37.0x

Twins, both liveborn

$\mathrm{Z37.2x}$

Twins, one liveborn one stillborn

$237.3 x$

Other multiple, all liveborn

Z37.5x

Other multiple, some liveborn

Z37.6x

Unspecified

Z37.9x

59515

59618

Liveborn infants consuming healthcare

Single liveborn

Z38.0x-

Z38.2x

Twin liveborn

$Z 38.3 x-$

Z38.5x

Other multiple liveborn Z38.6x-

59622

Encounter for full-term uncomplicated delivery

Z38.8x

O80x

Encounter for cesarean delivery without indication

O82x

ICD-10-PCS procedure

Extraction of POC, Classical, Open Approach

10D00Z0

Extraction of POC, Low Cervical, Open Approach

10D00Z1

Extraction of POC, Extraperitoneal, Open Approach

10D00Z2

Extraction of POC, Low Forceps, Via Opening

$10 \mathrm{D07Z3}$

Extraction of POC, Mid Forceps, Via Opening

10D07Z4

Extraction of POC, High Forceps, Via Opening

$10 \mathrm{D} 07 \mathrm{Z5}$

10D07Z6 Opening

Extraction of POC, Int Version, Via Opening

10D07Z7

Extraction of Products of Conception, Other, Via

10D07Z8

V30.xx Opening

Delivery of Products of Conception, External Approach 
Table 7 Codes to identify delivery (Continued)

\author{
2. Codes to identify cesarean delivery \\ CPT codes
}

Routine obstetric care including antepartum care,

cesarean delivery, and postpartum care

Cesarean delivery only

59514

Cesarean delivery only; including postpartum care

Routine obstetric care including antepartum care, cesarean delivery, and postpartum care, following attempted vaginal delivery after previous cesarean delivery

Cesarean delivery only, following attempted vaginal delivery after previous cesarean delivery;

Cesarean delivery only, following attempted vaginal delivery after previous cesarean delivery; including postpartum care

\section{ICD-9-CM diagnosis}

Single liveborn, born in hospital, delivered by cesarean delivery

Twin, mate liveborn, born in hospital, delivered by cesarean delivery

Twin, mate stillborn, born in hospital, delivered by cesarean delivery

Twin, unspecified whether mate stillborn or liveborn, born in hospital, delivered by cesarean delivery

Other multiple, mates all liveborn, born in hospital, delivered by cesarean delivery

Other multiple, mates all stillborn, born in hospital, delivered by cesarean delivery

Other multiple, mates liveborn and stillborn, born in hospital, delivered by cesarean delivery

Other multiple, unspecified whether mates stillborn or liveborn, born in hospital, delivered by cesarean delivery

Liveborn infant, unspecified whether single, twin, or multiple, born in hospital, delivered by cesarean

Cesarean delivery, without mention of indication

\section{ICD-9-CM procedure}

Cesarean section and removal of fetus

\section{ICD-10-CM diagnosis}

Single liveborn infant, delivered by cesarean

Twin liveborn infant, delivered by cesarean

Triplet liveborn infant, delivered by cesarean

Quadruplet liveborn infant, delivered by cesarean

Z38.64

Quintuplet liveborn infant, delivered by cesarean

Z38.66

Z38.69

Other multiple liveborn infant, delivered by cesarean

$\mathrm{O} 82 \mathrm{x}$

Encounter for cesarean delivery without indication

ICD-10-PCS procedure

Extraction of POC, Classical, Open Approach

10D00Z0

Extraction of POC, Low Cervical, Open Approach

10D00Z1

Extraction of POC, Extraperitoneal, Open Approach
Table 8 Codes to identify neonatal hyperbilirubinemia

\begin{tabular}{ll}
\hline Description & ICD- ICD-10-CM \\
& 9-CM
\end{tabular}

Hemolytic disease of fetus or newborn due $773.0 \quad$ P55.0

to $\mathrm{Rh}$ isoimmunization

Hemolytic disease of fetus or newborn due 773.1 P55.1

to $A B O$ isoimmunization

Hemolytic disease of fetus or newborn due 773.2 P55.8, P55.9

to other and unspecified isoimmunization

Kernicterus of fetus or newborn due to $\quad 773.4 \quad$ P57.0

isoimmunization

$\begin{array}{lll}\text { Perinatal jaundice from hereditary } & 774.0 & \text { P58.8 }\end{array}$

hemolytic anemias

Perinatal jaundice from other excessive $\quad 774.1 \quad$ P58.0, P58.2, P58.3,

hemolysis

P58.41, P58.5, P58.8

Kernicterus of fetus or newborn not due to $774.7 \quad$ P57.8

isoimmunization

Rh isoimmunization of newborn $\quad 794 \quad$ P55.0

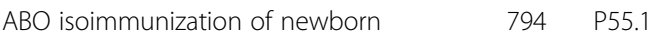

Other hemolytic diseases of newborn $\quad 793 \quad$ P55.8

Hemolytic disease of newborn, unspecified 793 P55.9

Kernicterus due to isoimmunization $\quad 793 \quad$ P57.0

$\begin{array}{lll}\text { Other specified kernicterus } & 793 & \text { P57.8 }\end{array}$

Kernicterus, unspecified $\quad 793 \quad$ P57.9

$\begin{array}{lll}\text { Neonatal jaundice due to bruising } & 794 & \text { P58.0 }\end{array}$

$\begin{array}{lll}\text { Neonatal jaundice due to bleeding } & 794 & \text { P58.1 }\end{array}$

$\begin{array}{lll}\text { Neonatal jaundice due to other specified } \quad 794 & \text { P58.8 }\end{array}$

excessive hemolysis

$\begin{array}{lll}\text { Neonatal jaundice due to excessive } & 794 & \text { P58.9 }\end{array}$

hemolysis, unspecified 
Table 9 Codes to identify NICU admission, gestational age estimation and newborn clinical conditions

\begin{tabular}{l} 
Descriptions \\
\hline Neonatal Intensive Care Unit (NICU) \\
Level 1 \\
Level 2 \\
Level 3 \\
Level 4 \\
Prenatal tests and preterm codes for gestational age estimation \\
Oral glucose tolerance test (OGTT) \\
Alpha-fetoprotein (AFP) \\
Inhibin-A (IHA) \\
Ultrasound, pregnant uterus, real time with image documentation, \\
fetal and maternal evaluation plus detailed fetal anatomic \\
examination \\
Pregnancy-associated protein plasma-A (PAPP-A) \\
Chorionic villus sampling \\
Preterm 35 -37 weeks \\
Preterm < 35 weeks
\end{tabular}

Preterm unspecified

Newborn clinical conditions

Birth trauma and/or hemorrhage (associated with the development of potential jaundice from bleeding)

Polycythemia neonatorum

Neonatal hematemesis and melena due to swallowed maternal blood

Breech delivery and extraction affecting fetus or newborn

Forceps delivery affecting fetus or newborn

Delivery by vacuum extractor affecting fetus or newborn

Other malpresentation, malposition, and disproportion during labor and delivery affecting fetus or newborn

Newborn neurodevelopmental disorders

Developmental delay

Cognitive disorders (nonspecific)

Language disorder (or speed disorder, dysarthria)

Motion dysfunction

ICD-9-CM

ICD-10-CM

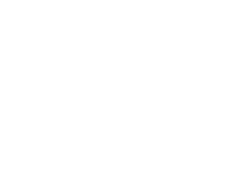

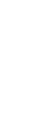

Revenue: 171

Revenue: 172

Revenue: 173

Revenue: 174

$82950,82951,82952$

82105

86336

$76801,76802,76805,76810$

$76811,76812,76813,76814$,

$76815,76816,76817,76818,76819,76820$

84163

59015
765.28

$765.21,765.22$

$765.23,765.24$

$765.25,765.26$

765.27

765.20,

$644.2 x$

864.00-

864.05,864.09,

$865.00-$

865.04,865.09,

$767.0,767.11,770.3$

$772.10-772.14$

$772.2-772.9$

776.4

763.0

763.2

763.3

763.1

299.xx, 315.8,

315.9, 330.8

$310.9 x$

$315.3 x$

$315.4 x, 781.2$,

719.7,
P78.2X

P07.38, P07.39

P07.21, P07.22,

P07.23, P07.24,

P07.25, P07.26,

P07.31, P07.32,

P07.33, P07.34,

P07.35, P07.36,

P07.37

P07.20, P07.30,

P07.0x, P07.1X,

O60.1x

P10x,P12.0x,P12.1X P12.3X,P15.0x,

P15.1X,P26x,

P52X, P53x, P54X

\section{P61.1x}

P03.0x

P03.2x

P03.3x

P03.1X

F84x, F88x, F89x

F09x

F80x

F82x, R26x, R27x 
Table 9 Codes to identify NICU admission, gestational age estimation and newborn clinical conditions (Continued)

\begin{tabular}{|c|c|c|c|}
\hline Descriptions & ICD-9-CM & ICD-10-CM & CPT/ Revenue codes \\
\hline & 781.3, & & \\
\hline Cerebral palsy & 343.x, 333.71, & G80x & \\
\hline Abnormal behavior & $\begin{array}{l}\text { 314.x, 312.x, } \\
\text { 309.21, 313.x } \\
\text { 307.x }\end{array}$ & F90x-F98x & \\
\hline Bilirubin encephalopathy (kernicterus spectrum disorder) & $773.4,774.7$ & P57x & \\
\hline Encephalopathy & $348.3 x$ & G93.4x & \\
\hline Hearing loss & $\begin{array}{l}\text { 389.xx, 388.01 } \\
388.2388 .11\end{array}$ & H90x, H91x & \\
\hline Vision loss & $368 . x x$ & H53x & \\
\hline
\end{tabular}

\section{Abbreviations}

AAP: American Academy of Pediatrics; Cl: Confidence Interval; GA: Gestational age; HCRU: Healthcare resource utilization; HDN: Hemolytic disease of the newborn; ICD-9/10-CM: The international classification of diseases, 9/10th Revision, clinical modification; ICD-9-CM/10-PCS: The international classification of diseases, 9/10th Revision, procedure coding system; IVlg: Intravenous immunoglobulin; KSDs: Kernicterus spectrum disorders; NHB: Neonatal hyperbilirubinemia; NICU: Neonatal intensive care unit; RBC: Red blood cell; SD: Standard deviation; TSB: Total serum bilirubin

\section{Acknowledgements}

The authors thank B. Bernard Tulsi for writing and editorial support and Nianya Liu for data programming and querying. We also would like to thank Dr. Joseph Singer, Chief Medical Officer, for providing clinical input and perspectives to the study. Thanks to Dr. Holly Romero, Andrew Rava, Ruixin Tan, and all reviewers of the protocol or manuscript for their helpful feedback. All errors or omissions remain the responsibility of the authors.

\section{Funding}

The study was funded by Mallinckrodt Pharmaceuticals. Authors T-CY, NR, and $E A B$, employees of Mallinckrodt at the time of the study, participated in study design, data interpretation, and revising different versions of this article, and approved it for submission.

\section{Availability of data and materials}

The corresponding author has direct access to the dataset used for this study, clarification could be provided upon reasonable request.

\section{Authors' contributions}

All the authors contributed substantially to the conceptualization and design of this study. Authors CN, SZ, XZ and HT, employees of HealthCore, engaged in data acquisition and analysis. Authors T-CY, NR, and EAB, were/are employees of Mallinckrodt Pharmaceuticals, along with all the other authors $\mathrm{CN}, \mathrm{NR}, \mathrm{SZ}, \mathrm{XZ}$, and HT were involved in study design, data interpretation and participated in drafting and revising the different versions of this article. The final version of the article was approved by all authors.

\section{Ethics approval and consent to participate}

This non-experimental observational study was exempt from Institutional Review Board (IRB) informed consent stipulations. The researchers accessed a limited dataset without individual enrollee identifiers, and only summary statistics were reported. This work complied with all relevant provisions of the US federal Health Insurance Portability and Accountability Act of 1996 (HIPAA)

\section{Consent for publication}

Not applicable. The study does not contain data from any individual person.

\section{Competing interests}

The authors declare the following: T-CY is an employee and a stockholder of Mallinckrodt Pharmaceuticals, Bedminster, New Jersey 07921, USA. NR was an employee and is a stockholder of Mallinckrodt Pharmaceuticals, Bedminster, New Jersey 07921, USA. EAB is an employee of Mallinckrodt Pharmaceuticals,
Bedminster, New Jersey 07921, USA. CN, SZ, XZ, HT are employees of HealthCore, Inc Wilmington, Delaware 19,801, USA whose activities on research projects are funded by various pharmaceutical/biotech/medical device companies.

\section{Publisher's Note}

Springer Nature remains neutral with regard to jurisdictional claims in published maps and institutional affiliations.

\section{Author details}

${ }^{1}$ Mallinckrodt Pharmaceuticals, Bedminster, NJ 07921, USA. ${ }^{2}$ HealthCore, Inc., An Independent Subsidiary of Anthem, Inc, Wilmington, DE 19801, USA.

Received: 10 August 2018 Accepted: 22 January 2019

Published online: 11 February 2019

\section{References}

1. Cohen RS, Wong RJ, Stevenson DK. Understanding neonatal jaundice: a perspective on causation. Pediatr Neonatol. 2010;51(3):143-8.

2. Ullah S, Rahman K, Hedayati M. Hyperbilirubinemia in neonates: types, causes, clinical examinations, preventive measures and treatments: a narrative review article. Iran J Public Health. 2016;45(5):558-68.

3. Kaplan M, Hammerman C. Understanding and preventing severe neonatal hyperbilirubinemia: is bilirubin neurotoxity really a concern in the developed world? Clin Perinatol. 2004:31(3):555-75 x.

4. Porter ML, Dennis BL. Hyperbilirubinemia in the term newborn. Am Fam Physician. 2002:65(4):599-606.

5. Woodgate P, Jardine LA. Neonatal jaundice. BMJ Clin Evid. 2011;2011.

6. Woodgate $P$, Jardine LA. Neonatal jaundice: phototherapy. BMJ Clin Evid. 2015;2015.

7. Smitherman H, Stark AR, Bhutani VK. Early recognition of neonatal hyperbilirubinemia and its emergent management. Semin Fetal Neonatal Med. 2006;11(3):214-24.

8. Memon N, Weinberger Bl, Hegyi T, Aleksunes LM. Inherited disorders of bilirubin clearance. Pediatr Res. 2016;79(3):378-86.

9. Murray NA, Roberts IA. Haemolytic disease of the newborn. Arch Dis Child Fetal Neonatal Ed. 2007;92(2):F83-8.

10. American Academy of Pediatrics Subcommittee on $\mathrm{H}$. Management of hyperbilirubinemia in the newborn infant 35 or more weeks of gestation. Pediatrics. 2004:114(1):297-316.

11. Maisels MJ, Watchko JF, Bhutani VK, Stevenson DK. An approach to the management of hyperbilirubinemia in the preterm infant less than 35 weeks of gestation. J Perinatol. 2012;32(9):660-4.

12. Amin SB, Bhutani VK, Watchko JF. Apnea in acute bilirubin encephalopathy. Semin Perinatol. 2014;38(7):407-11.

13. Muchowski KE. Evaluation and treatment of neonatal hyperbilirubinemia. Am Fam Physician. 2014:89(11):873-8.

14. Watchko JF, Tiribelli C. Bilirubin-induced neurologic damage--mechanisms and management approaches. N Engl J Med. 2013;369(21):2021-30.

15. Wasser T, We B, Ycas J, Tunceli O. Applying Weighting Methodologies to a Commercial Database to Project US Census Demographic Data. Am J Account Care. 2015;3:33-8. 
16. Wallace $A E$, Le BK, Selvam $N$, et al. Estimation of gestional age at birth using claims-based algorithms in a commercially insured US population. Presented at the International Society for Pharmacoepidemiolkogy (ISPE) 32nd international conference on Pharmacoepidemiology (ICPE) and therapeutic risk management. 2016;Dublin, Ireland Astract available at https:/onlinelibrary. wiley.com/doi/full/10.1002/pds.4070\#. Accessed 23 Apr 2018.

17. Consumer Price Index for Medical Care. Available at https://www.hrsa.gov/ get-health-care/affordable/hill-burton/cpi.html. Accessed 23 Apr 2018.

18. United States Department of Health and Human Services (US DHHS), Centers for Disease Control and Prevention (CDC), National Center for Health Statistics (NCHS), Division of Vital Statistics, Natality public-use data 2007-2016, on CDC WONDER Online Database, February 2018. Available at http://wonder.cdc.gov/natality-current.html . Accessed 29 May 2018.

19. Curtin LR, Klein RJ. Direct standardization. Statistical Notes. 1995;Available at https:/www.cdc.gov/nchs/data/statnt/statnt06rv.pdf . Accessed 29 May 2018.

20. Bhutani VK, Stark AR, Lazzeroni LC, Poland R, Gourley GR, Kazmierczak S, et al. Predischarge screening for severe neonatal hyperbilirubinemia identifies infants who need phototherapy. J Pediatr. 2013;162(3):477-82 e1.

21. Burke BL, Robbins JM, Bird TM, Hobbs CA, Nesmith C, Tilford JM. Trends in hospitalizations for neonatal jaundice and kernicterus in the United States, 1988-2005. Pediatrics. 2009:123(2):524-32.

22. Wagle S, Deshpande PG. Hemolytic Disease of the Newborn. Medspace. 2017.

23. Chang PW, Kuzniewicz MW, McCulloch CE, Newman TB. A Clinical Prediction Rule for Rebound Hyperbilirubinemia Following Inpatient Phototherapy. Pediatrics. 2017:139(3).

24. Bhutani VK, Johnson LH, Schwoebel A, Gennaro S. A systems approach for neonatal hyperbilirubinemia in term and near-term newborns. J Obstet Gynecol Neonatal Nurs. 2006;35(4):444-55.

25. Eggert LD, Wiedmeier SE, Wilson J, Christensen RD. The effect of instituting a prehospital-discharge newborn bilirubin screening program in an 18hospital health system. Pediatrics. 2006;117(5):e855-62.

26. Atkinson LR, Escobar GJ, Takayama Jl, Newman TB. Phototherapy use in jaundiced newborns in a large managed care organization: do clinicians adhere to the guideline? Pediatrics. 2003:111(5 Pt 1):e555-61.

27. Maisels MJ, Kring E. Length of stay, jaundice, and hospital readmission. Pediatrics. 1998:101(6):995-8.

28. Usatin D, Liljestrand P, Kuzniewicz MW, Escobar GJ, Newman TB. Effect of neonatal jaundice and phototherapy on the frequency of first-year outpatient visits. Pediatrics. 2010;125(4):729-34.

29. Truven Health Analytics. The cost of having a baby in the United Sates. Marketscan study prepared for childbirth connection, Catalyst for Payment Reform and Center for Healthcare Quality and Payment Reform; 2013. Available at https://transform.childbirthconnection.org/wpcontent/uploads/2013/01/Cost-of-Having-a-Baby-Executive-Summary.pdf. (Accessed 29 May 2018).

30. Ku L, Broaddus M. Public and private health insurance: stacking up the costs. Health Aff (Millwood). 2008:27(4):w318-27.

31. Brooks JC, Fisher-Owens SA, Wu YW, Strauss DJ, Newman TB. Evidence suggests there was not a "resurgence" of kernicterus in the 1990s. Pediatrics. 2011;127(4):672-9.

32. Bhutani VK, Johnson L. Kernicterus in the 21st century: frequently asked questions. J Perinatol. 2009;29(Suppl 1):S20-4.

33. Romero HM, Ringer C, Leu MG, Beardsley E, Kelly K, Fesinmeyer MD, et al. Neonatal Jaundice: Improved Quality and Cost Savings After Implementation of a Standard Pathway. Pediatrics. 2018.

34. Hudak ML, Rosenfeld W, Ruiz N. Tin Mesoporphyrin (SnMP) as Adjunctive Treatment to Phototherapy (PT) in Neonates with Hyperbilirubinemia (HB) and Hemolysis. Pediatric Academic Societies Meeting; Toronto, ON, CanadaMay 5-8, 2018.

35. Bhutani VK, Poland R, Meloy LD, Hegyi T, Fanaroff AA, Maisels MJ. Clinical trial of tin mesoporphyrin to prevent neonatal hyperbilirubinemia. J Perinatol. 2016;36(7):533-9.

36. Beyrer J, Nolot S, Haldane D, Johnston J. Developing an ICD-10-CM Version of Charlson Comorbidities for United States Real-world Healthcare Data. ISPOR 22nd Annual International Meeting; Boston, MA; May 20-24, 2017. 2017.

\section{Ready to submit your research? Choose BMC and benefit from:}

- fast, convenient online submission

- thorough peer review by experienced researchers in your field

- rapid publication on acceptance

- support for research data, including large and complex data types

- gold Open Access which fosters wider collaboration and increased citations

- maximum visibility for your research: over $100 \mathrm{M}$ website views per year

At BMC, research is always in progress.

Learn more biomedcentral.com/submissions 